\title{
Educational intervention for liver transplantation candidates ${ }^{1}$
}

\author{
Karina Dal Sasso Mendes² \\ Orlando de Castro e Silva Junior ${ }^{3}$ \\ Luciana da Costa Ziviani ${ }^{4}$ \\ Fabiana Murad Rossin ${ }^{4}$ \\ Márcia Maria Fontão Zago ${ }^{5}$ \\ Cristina Maria Galvão ${ }^{6}$
}

\begin{abstract}
Objective: The objective in this study was to analyze candidates' knowledge on the liver transplantation process before and after putting in practice an educational intervention. Method: A quasi-experimental, one-group pretest-posttest research design was adopted. The final sample included 15 subjects. Research data were collected between January and March 2010 in three phases, which were: pretest, implementation of the educational intervention (two meetings) and posttest. Results: The results evidenced significant cognitive gains after the intervention, with improvements in the participants' performance. Conclusions: The research presents evidence that putting in practice a patient education strategy can enhance candidates' knowledge on the liver transplantation process and consequently contribute to a successful treatment.
\end{abstract}

Descriptors: Nursing; Liver Transplantation; Teaching; Learning.

\footnotetext{
${ }^{1}$ Paper extracted from Doctoral Dissertation "O processo ensino-aprendizagem para o candidato ao transplante de fígado" presented to Escola de Enfermagem de Ribeirão Preto, Universidade de São Paulo, WHO Collaborating Centre for Nursing Research Development, Ribeirão Preto, SP, Brazil.

2 PhD, RN, Escola de Enfermagem de Ribeirão Preto, Universidade de São Paulo, WHO Collaborating Centre for Nursing Research Development Ribeirão Preto, SP, Brazil.

${ }^{3}$ PhD, Full Professor, Faculdade de Medicina de Ribeirão Preto, Universidade de São Paulo, Ribeirão Preto, SP, Brasil.

${ }^{4}$ Master's Students, Escola de Enfermagem de Ribeirão Preto, Universidade de São Paulo, WHO Collaborating Centre for Nursing Research Development, Ribeirão Preto, SP, Brazil. RN, Hospital das Clínicas, Faculdade de Medicina de Ribeirão Preto, Universidade de São Paulo, Ribeirão Preto, SP, Brasil.

5 PhD, Associate Ptrofessor, Escola de Enfermagem de Ribeirão Preto, Universidade de São Paulo, WHO Collaborating Centre for Nursing Research Development, Ribeirão Preto, SP, Brazil.

${ }^{6}$ PhD, Full Professor, Escola de Enfermagem de Ribeirão Preto, Universidade de São Paulo, WHO Collaborating Centre for Nursing Research Development, Ribeirão Preto, SP, Brazil.
} 


\section{Introduction}

Nurses are the health team members who spend most time with the patient, giving them an essential role as educators in the different learning needs diseases demand(1). Therefore, these professionals' scientific background is relevant with a view to putting in practice effective strategies to promote changes in patients' behavior, attitudes and lifestyles.

People with chronic liver failure need nursing interventions to support the required lifestyle changes, so as to prevent and control disease progress. At some moment in their disease, patients will be confronted with acute aggravations, such as refractory ascites, hepatic encephalopathy and digestive hemorrhage, which will demand professional health care delivery in the hospital context. Nursing care is focused on promoting the development of patient skills for disease self-management, which will only be possible if nurses and the multidisciplinary team use educational interventions ${ }^{(2)}$.

In Brazilian literature, nurses involved in organ transplantation programs have hardly explored the patient teaching-learning process as a theme for knowledge production, which motivated the accomplishment of this research ${ }^{(3)}$.

Patient education can be defined as the process through which patient achieve an understanding of their own physical condition and accomplish self-care through the use of different experiences and resources. The goal of education is to enable patients not only to understand their current health condition, but also to be capable of making healthcare-related decisions ${ }^{(4)}$.

Among the benefits of the health education process for patients, increased satisfaction and quality of life are highlighted, as well as improved care continuity at home, reduction of anxiety levels and possible complications, enhanced adherence to the proposed treatment plan, maximization of independence and empowerment ${ }^{(4-5)}$.

Patients who need solid organ transplantation suffer from a chronic condition, which by itself entails risks and health problems. Thus, patients who are capable of understanding the transplantation can also change their living experience. They need to learn how to deal with new drugs, take them for the rest of their lives, besides adhering to lifestyle changes, including hygiene practices, infection prevention, monitoring of the new organ's functioning, body image changes, adaptation to mood and energy level swings, professional issues, among others ${ }^{(6)}$.
In this study, to plan and put in practice the teaching-learning process involving liver transplantation candidates, the theoretical premises proposed by experts in the area were used(7). This theory is based on the information processing model, considered important to develop health-related learning.

Learning is defined as the process that allows individuals to permanently change their behavior. It happens when people respond to and receive stimuli from their external environment. When observing changes in learners' performance, this shows that learning has taken place(7).

In view of the above, the aim of this research was to analyze candidates' knowledge on the liver transplantation process before and after putting in practice an educational intervention.

\section{Methods}

A quasi-experimental, one-group, pretest-posttest design was adopted. The study was undertaken at a general public hospital in the interior of São Paulo State, which offers a liver transplantation program registered in the National Transplantation System. The study population consisted of subjects with a technical liver registration, with deceased donor, awaiting surgery, totaling 77 patients during the year the research was conducted.

As regards the selection criteria, patients were considered who were 18 years of age or older, MELD (Model for End-stage Liver Disease - model that indicates the severity of the liver disease) classification between six (least severe) and 25 (most severe); in clinical conditions to receive the educational intervention and literate (reading and writing) to permit the completion of the data collection instrument.

Subjects who expressed or displayed an evolution in their liver disease that negatively affected their participation in the educational intervention, recovered their liver function or died during the data collection period were excluded $(n=22)$.

During the data collection period, which took three consecutive months (from January to March 2010), the researchers invited all patients who complied with the established selection criteria to participate in the educational intervention $(n=55)$. The patients were invited 30 days before the intervention, characterizing a convenience sample. Out of 55 patients, 15 attended the first meeting. In view of the difficulties the patients expressed to participate in the study, a second group 
was planned. On that occasion, four additional patients attended, totaling 19 patients. During the interval between one meeting and the second, however, four patients were unable to conclude the educational intervention for the following reasons: one patient was submitted to a liver transplantation, one died, one was going through an acute phase of hepatic encephalopathy and the other did not attend without any justification. Therefore, 15 patients participated in this research.

To plan the educational intervention, a teaching plan was elaborated. One of the researchers was responsible for putting the intervention in practice. The planning and execution of the educational intervention was based on learning development theory ${ }^{(7)}$, on the teaching-learning model for organ transplantation patients $^{(8)}$ and on the diagnostic assessment of patients' informational needs, developed in an earlier study.

The educational intervention took place in an indoor auditorium near the Liver Transplantation Unit, which offers multimedia equipment, a big screen, 76 places and air-conditioning to enhance the research participants' comfort and wellbeing.

The educational intervention was put in practice during two meetings. In the first, the following topics were addressed: general view of the organ for transplantation and historical aspects of liver transplantation; awaiting a new organ; the organ distribution system and the liver donor; the day of the transplantation, surgery and anesthesia.

During the second meeting, the topics addressed were: hospitalization period; medication used after the transplantation; post-transplant complications; quality of life after the transplantation and care needed across the lifetime. At the end of the second meeting, a discussion was held between the candidates and post-transplant patients, which took approximately one hour. After each meeting, a snack was served in accordance with the transplantation team nutritionist's recommendations.

In total, the educational intervention took six hours, with four hours of dialogued class and two hours of discussion (first and second meetings). To develop the intervention, active teaching-learning strategies were used, encouraging discussions between the patients (students) and the nurse (facilitator) as contents were developed.

To assess patients' knowledge, an instrument was elaborated with 17 multiple-choice questions, addressing the main phases of the liver transplantation process (pre, intra and post-transplant). The elaboration of the instrument was based on publications in the area( ${ }^{(8-9)}$ and on educational materials from the International Transplant Nurses Society focused on patient teaching ${ }^{(10)}$, as well as on the information folder for patients enrolled in the liver transplantation program at the place of study.

The instrument was submitted to face and content validation, involving seven judges. The selected judges were three nurses and two physicians active in liver transplantation, one faculty member experienced in instrument validation and one post-transplant patient. Inter-rater agreement on the instrument items exceeded $80 \%$. The researchers accepted their suggestions, related to the presentation form of the instrument.

Research data were collected in three phases, which were: pretest, application of patient knowledge assessment instrument and completion of the informed consent form, educational intervention (two meetings) and post-test, application of the patient knowledge assessment instrument after the educational intervention.

Descriptive statistics were used for data analysis. Data on quantitative variables were summarized as arithmetic means and their respective standard deviations. Parametric (Student's t-test and Pearson's correlation coefficient) and non-parametric (KruskalWallis test and Spearman's rank correlation coefficient) statistical methods were applied. Data on qualitative variables were summarized in the form of percentages. To compare data on a given dichotomous variable between two groups ( $2 \times 2$ contingency tables), Fisher's exact test was used. GraphPad InStat 3.05 software (GraphPad Software, Inc.) was employed for all descriptive analyses. Significance was set at $5 \%(\alpha=0.05)$. Approval for this study was obtained from the Research Ethics Committee at the University of São Paulo at Ribeirão Preto Medical School Hospital das Clínicas (process 12953/2008).

\section{Results}

Out of 19 patients who participated in the first educational meeting, $16(84.21 \%)$ were men and three (15.79\%) women. The mean age was 52.84 years $(\mathrm{SD}=7.57) ; 14(73.68 \%)$ were born in urban and five $(26.32 \%)$ in rural areas. Only four patients $(21.05 \%)$ were working, while the remaining 15 (78.95\%) were on a leave of absence. The mean education corresponded to 7.53 years $(S D=3.82)$, ranging between three and 15. Thirteen $(68.42 \%)$ candidates held a primary, three $(15.79 \%)$ a secondary and three $(15.79 \%)$ a higher education degree.

As regards patients' clinical conditions, the mean 
MELD score was $15.16 \quad(S D=2.06)$, four subjects $(21.05 \%)$ suffered from alcohol-caused liver cirrhosis, $13(68.42 \%)$ from hepatitis-caused liver cirrhosis (five of which also associated with alcohol consumption), one (5.26\%) from cryptogenic liver cirrhosis and the other (5.26\%) from nonalcoholic steatohepatitis (NASH). The mean waiting time for the liver transplant was $1,247.53$ days $(S D=661.78)$.

In the application of the knowledge assessment instrument on the transplantation process before the educational intervention, the mean correct answer rate was $10.37(S D=3.06)$ and the mean error rate 6.63 $(S D=3.06)$, with a global correct answer percentage of $60.99 \%$. The number of correct answers ranged between three and 15 .

Data analysis showed that patients up to 55 years of age gave more correct answers (67.97\%) when compared to younger patients $(54.71 \%)$, although this difference was not statistically significant $(p=0.1103$, Student's t-test). Pearson's correlation analysis only revealed a negative correlation trend between the percentage of correct answers and age (Pearson's correlation coefficient: $r=-0.3310 ; p=0.1663)$. A significant positive correlation was found between the percentage of correct answers and years of education (Spearman's rank correlation: $r_{s}=0.4899 ; p=0.0332$ ). A ranking of the subjects according to education level, however, showed lower correct answer rates (57.01\%) for candidates with a primary education degree only when compared to candidates with secondary, higher or post-graduate degrees $(69.61 \%)$, although this difference was not significant $(p=0.1619$, Student's t-test).

No correlation was found between waiting times and patients' correct answer rates (Pearson's correlation coefficient: $r=-0.0298 ; p=0.9036)$, with a mean correct answer rate of $61.18 \%$ for patients with up to two years of waiting time, against $62.57 \%$ for patients who had been waiting between two and five years and $54.90 \%$ for patients with more than five years of waiting time ( $p=0.9900$, Kruskal-Wallis test). When comparing the subjects according to the severity of their liver disease, that is, according to their MELD scores, patients scoring up to 14 points showed $53.78 \%$ of correct answers, against $65.2 \%$ for candidates scoring more than 14 points ( $p=0.1899$, Student's t-test). Despite this difference, the MELD showed no correlation with patients' correct answer scores (Pearson's correlation coefficient: $r=0.0343 ; p=0.8891$ ).

As regards correct answers rates on the knowledge assessment instrument about the liver transplantation process, questions with higher rates were related to factors contributing to a successful transplantation $(100 \%)$, followed by questions on transplantation candidates' responsibilities (94.74\%) and the postoperative period, specifically hospitalization $(89.47 \%)$. The lowest correct answer rates were for: questions on the immunosuppressants used after the liver transplantation (10.53\%), on the care needed after the liver transplantation (15.79\%), complications after the liver transplantation (21.05\%) and quality of life aspects after the transplant (31.58\%). All four questions were related to the postoperative period.

In general, patients scored higher on questions about the preoperative period (70.40\%), followed by the intraoperative $(68.42 \%)$ and postoperative periods (44.74\%). No statistically significant difference was found in correct answer rates among the three periods ( $p=0.4560$, Kruskal-Wallis test).

It is highlighted that, during the interval between one meeting and the second (the intervention involved two meetings), one patient was submitted to a liver transplantation, one died, one was going through an acute phase of hepatic encephalopathy and the other did not attend without any justification, totaling a final research sample of 15 patients.

In the analysis of correct answers to the 17 questions in the knowledge assessment instrument on the transplantation process before and after the educational intervention, a statistically significant difference is again observed, on the one-tailed test $(p=0.0043$, Student's one-tailed t-test for paired samples) as well as on the two-tailed test $(p=0.0086$, Student's one-tailed t-test for paired samples). In general, only questions on the postoperative period demonstrated a significant increase in the correct answer rate (Fisher's one-tailed exact test). In addition, none of the questions showed $100 \%$ of correct answers after the educational intervention.

Higher correct answer rates after the educational intervention were related to: question on the importance of the liver $(93.33 \%)$, related to the waiting time for transplantation (93.33\%), signs of worsening in the liver disease $(93.33 \%)$ and the day of the liver transplantation (93.33\%). The highest error rate $(66.67 \%)$ was related to the question on the care needed after the liver transplantation. As regards correct answers on questions related to the perioperative period, the highest mean correct answer rate $(74.17 \%$ before and $82.50 \%$ after the intervention) was for questions on the preoperative 
period, followed by questions on the intraoperative (71.11\% before and $80 \%$ after the intervention) and postoperative periods (45.56\% before and $68.89 \%$ after the intervention).

In the general analysis of correct answer rates, a global increase by 2.13 questions $(12.55 \%)$ was observed after the intervention, leading to a mean performance improvement equivalent to $19.75 \%$ (ratio between global increase - $12.55 \%$ - and correct answer rate on knowledge assessment instrument before the intervention $-63.53 \%$ ).
The correct answer rate increased from $63.53 \%$ before the intervention to $76.08 \%$ afterwards. This difference was statistically significant on the onetailed ( $p=0.0117$, Student's one-tailed t-test for paired samples) as well as on the two-tailed test $(p=0.0234$, Student's one-tailed t-test for paired samples). The number of patients who correctly answered less than $80 \%$ of the questions dropped from 13 (86.67\%) before the intervention to merely six (40\%) after the intervention (Table 1).

Table 1 - Patient distribution according to correct answer rates on the knowledge assessment instrument applied before and after the educative intervention for liver transplantation candidates, Ribeirão Preto, SP, Brazil, 2010

\begin{tabular}{|c|c|c|c|c|c|c|c|c|}
\hline \multirow{2}{*}{ Patients } & \multicolumn{2}{|c|}{ Before the intervention $(n=15)$} & \multirow{2}{*}{$\begin{array}{c}\% \text { of Correct } \\
\text { Answers }\end{array}$} & \multicolumn{2}{|c|}{ After the Intervention $(n=15)$} & \multirow{2}{*}{$\begin{array}{c}\% \text { of Correct } \\
\text { Answers }\end{array}$} & \multirow{2}{*}{$\begin{array}{l}\text { Performance } \\
\text { Variation (\%) }\end{array}$} & \multirow{2}{*}{$\begin{array}{l}p \text { (Fisher's } \\
\text { one-tailed } \\
\text { exact test) }\end{array}$} \\
\hline & Correct & Incorrect & & Correct & Incorrect & & & \\
\hline P.01 & - & - & - & - & - & - & - & - \\
\hline P.02 & - & - & - & - & - & - & - & - \\
\hline P.03 & 3 & 14 & 17.65 & 6 & 11 & 35.29 & +17.65 & 0.2192 \\
\hline P.04 & 8 & 9 & 47.06 & 9 & 8 & 52.94 & +5.88 & 0.5000 \\
\hline P.05 & 13 & 4 & 76.47 & 14 & 3 & 82.35 & +5.88 & 0.5000 \\
\hline P.06 & 13 & 4 & 76.47 & 16 & 1 & 94.12 & +17.65 & 0.1676 \\
\hline P.07 & 11 & 6 & 64.71 & 13 & 4 & 76.47 & +11.76 & 0.3540 \\
\hline P.08 & 14 & 3 & 82.35 & 14 & 3 & 82.35 & 0 & - \\
\hline P.09 & 10 & 7 & 58.82 & 7 & 10 & 41.18 & -17.65 & - \\
\hline P.10 & 13 & 4 & 76.47 & 11 & 6 & 64.71 & -11.76 & - \\
\hline P.11 & 15 & 2 & 88.24 & 17 & 0 & 100.00 & +11.76 & 0.2424 \\
\hline P.12 & 12 & 5 & 70.59 & 12 & 5 & 70.59 & 0 & - \\
\hline P.13 & 12 & 5 & 70.59 & 14 & 3 & 82.35 & +11.76 & 0.3440 \\
\hline P.14 & 10 & 7 & 58.82 & 14 & 3 & 82.35 & +23.53 & 0.1294 \\
\hline P.15 & 10 & 7 & 58.82 & 15 & 2 & 88.24 & +29.41 & 0.0558 \\
\hline P.16 & - & - & - & - & - & - & - & - \\
\hline P.17 & 12 & 5 & 70.59 & 15 & 2 & 88.24 & +17.65 & 0.1992 \\
\hline P.18 & 6 & 11 & 35.29 & 17 & 0 & 100.00 & +64.71 & $<0.0001$ \\
\hline P.19 & - & - & - & - & - & - & - & - \\
\hline Mean & 10.80 & 6.20 & 63.53 & 12.93 & 4.07 & 76.08 & +12.55 & - \\
\hline SD & 3.17 & 3.17 & 18.63 & 3.37 & 3.37 & 19.82 & 19.11 & - \\
\hline
\end{tabular}

\section{Discussion}

The main challenge to undertake this research was to recruit liver transplantation candidates for the educational intervention. In this study, besides a formal letter sent by mail, patients received telephone calls before the scheduled meetings. Also, the clinical conditions in which chronic liver conditions evolve intervene in an effective educational process. Despite using the available patient recruitment resources, a larger sample of candidates from the technical liver register could not be obtained.

As regards the candidates' performance on the research instrument, patients' knowledge on the factors contributing to successful transplantation and their responsibilities as candidates on the waiting list was noteworthy, with the highest correct answer rates. This shows that, despite patients' difficulties to adhere to the treatment proposed in organ transplantation programs, as reported in the literature ${ }^{(4,11-12)}$, patients are aware of what needs to be done to contribute to the success of their treatment. To illustrate the problem, in 2007, a study was published in Portugal, showing frequent nonadherence among liver transplantation receivers, with a mean prevalence of $25.28 \%{ }^{(13)}$.

When potential candidates are indicated for inclusion in the technical liver transplantation register, they need relevant information on the pre, intra and 
postoperative phases of the surgery. Data analysis of the knowledge assessment instrument indicated that the research participants knew more about the preoperative phase, to the detriment of the other phases, before and after the educative intervention. This result may reflect the candidates' experience until then, awaiting the surgery. Nevertheless, knowing what to expect in the future, in view of the complexity of liver transplantations, helps to raise patients and relatives' awareness and accountability for self-care, especially in the postoperative phase, when lifestyle changes are fundamental for treatment maintenance and success.

At a liver transplantation center in the United Kingdom, before patients are accepted on the waiting list, candidates and relatives/caregivers are invited to participate in a group education session, involving a team of transplant coordinator nurses. This session takes approximately two hours. Three months after the group session, a questionnaire is applied among the participants to assess the intervention. The authors concluded that the introduction of group sessions has helped to administer the time the team spends on patient teaching. A relevant finding is patients and relatives/caregivers' poor understanding about the transplantation process ${ }^{(14)}$.

In Spain, in a study undertaken to assess the efficacy of teaching-learning strategies for liver transplantation patients, the authors compared compliance with recommendations provided upon discharge from hospital between patients who only received oral information and those who received written and/or audiovisual information. In the research, a questionnaire was used to measure compliance with the treatment proposed upon hospital discharge, which addressed daily living habits, harmful habits, pharmacological treatment, use of preventive medicines (vaccines, sunscreen), physical exercise and emergency situations. The results demonstrated lower compliance with discharge recommendations among patients who had only received oral information when compared to patients who received written and/or audiovisual support in the educational process ${ }^{(15)}$. This study supports the present research findings, as audiovisual resources were used to put the educational intervention in practice, which in principle was effective in the study sample.

In Brazilian literature, there is a lack of studies on patient teaching in liver transplantation programs. In a study published in 2005 , the aim was to describe the learning outcomes of teaching strategies used with candidates awaiting liver transplantation. In that descriptive study, a knowledge questionnaire was applied, based on written information the candidates provided upon their inclusion in the technical register. The questionnaire was applied after an educational discussion, during which patients and relatives expressed their doubts and received further information on the transplantation process. The results showed that, on average, the global correct answer rate on the knowledge assessment questionnaire was about $80 \%$, with higher rates on questions related to the preoperative period ${ }^{(16)}$.

In 2007, in another Brazilian study, the efficacy of an interdisciplinary orientation group for liver transplantation candidates was assessed. A 17-item questionnaire was used to assess patients' opinions on the transplantation process. Group efficacy was assessed according to the correct answer rate before and after participating in the group. The intervention took place during a two-hour session. The results showed a $59 \%$ increase in the correct answer rate after the intervention ${ }^{(17)}$, although the study contained no indications as to how the questionnaire had been constructed and validated.

\section{Conclusions}

In view of the research objective, which was to analyze candidates' knowledge on the liver transplantation process before and after putting in practice an educative intervention, significant cognitive gains were verified after the intervention, with an average improvement by almost $20 \%$ in participants' performance. Statistical analysis showed $p<0.05$ for correct answer rates before and after the intervention.

Despite the small sample, until date, no study on the theme has been published in Brazilian literature. In conclusion, the educative intervention contributed to improve candidates' knowledge on the liver transplantation process.

Thus, the relevance of nurses' role is highlighted as educators in patient preparation, especially for complex surgical procedures like organ transplantations.

\section{References}

1. Kruzik N. Benefits of preoperative education for adult elective surgery patients. AORN J. 2009;90(3):381-7.

2. Grogan TA. Liver transplantation: issues and nursing care requirements. Crit Care Nurs Clin North Am. 2011;23(3):443-56.

3. Mendes KD, Galvão CM. Liver transplantation: evidence for nursing care. Rev. Latino-Am. Enfermagem. 2008;16(5):915-22 
4. Redman BK. Patient adherence or patient selfmanagement in transplantation: an ethical analysis. Prog Transplant. 2009;19(1):90-4.

5. Feldman-Stewart D, Brennenstuhl S, Brundage MD. A purpose-based evaluation of information for patients: an approach to measuring effectiveness. Patient Educ Couns. 2007;65(3):311-9.

6. Duma E. Liver transplantation. Nurs Stand. 2012;26(41):59-60.

7. Gagné RM. Princípios essenciais da aprendizagem para o ensino. Porto Alegre: Editora Globo; 1980. 175 p. 8. Ohler L. Patient education. In: Cupples SA, Ohler L. Transplantation nursing secrets. Philadelphia: Hanley \& Belfus; 2003. p. 305-12.

9. Gordon FD. 100 Questions and answers about liver transplantation: a Lahey Clinic guide. Sudbury: Jones and Bartlett Publishers; 2007. 199 p.

10. International Transplant Nurses Society (US). Liver transplant handbook: a guide for your health care after liver transplantation.[internet]. Pittsburg (US): International Transplant Nurses Society; 2007. [acesso 5 nov 2010]. Disponível em: http://www.itns. org/templates/itnsbasic/pdfs/ITNS_Liver_Transplant_ Booklet.pdf.

11. Telles-Correia D, Barbosa A, Mega I, Monteiro E. Adherence correlates in liver transplant candidates. Transplant Proc. 2009;41(5):1731-4.

12. De Geest S, Dobbels F. Transplantation: Increasing adherence to immunosuppression: a clinical priority. Nat Rev Nephrol. 2010;6(3):139-40.

13. Telles-Correia D, Barbosa A, Mega I, Barroso E, Monteiro E. Adesão nos doentes transplantados. Acta Med Port. 2007;20(1):73-85.

14. Ashcroft P. Adapting patient education for potential liver transplant recipients in a climate of chronic donor organ shortfall. Prog Transplant. 2009;19(1):59-63.

15. Cerezo MCM, Simón CS, Muñoz MP, Ramanillos MTA, Agustí IT, Canales PB. Estudio del proceso educativo en el paciente con trasplante hepático. Enferm Intensiva. 2001;12(2):58-65.

16. Sasso KD, Galvão CM, Castro-e-Silva O, França AVC. Liver transplantation: teaching strategies used with patients waiting for surgery. Rev. Latino-Am. Enfermagem. 2005;13(4):481-8.

17. Guimaro MS, Lacerda SS, Bacoccina TD, Karam CH, de Sa JR, Ferraz-Neto $\mathrm{BH}$, et al. Evaluation of efficacy in a liver pretransplantation orientation group. Transplant Proc. 2007;39(8):2522-4. 